\title{
Identification and elimination of false-positives in an ELISA-based system for qualitative assessment of glycoconjugate binding using a selection of plant lectins
}

\author{
Babak Afrough, Miriam V. Dwek, and Pamela Greenwell \\ University of Westminster, London, UK \\ BioTechniques 43:458-464 (October 2007) \\ doi $10.2144 / 000112554$
}

Systematic optimization of a lectin-based enzyme-linked immunosorbent assay (ELISA) procedure using a panel of 21 biotinylated plant lectins and a glycoprotein with defined glycosylation (i) identified blocking as a limiting step in solid phase sugar binding analysis and (ii) found Synblock to be a better alternative to bovine serum albumin (BSA) as blocking agent.

An experimental system was optimized to characterize the glycans of a mucin-like glycoprotein (1) by adsorption of glycoconjugate using a solid phase enzyme-linked immunosorbent assay (ELISA)-based method (2) with a selection of 21 plant lectins $(3,4)$. The glycoprotein utilized to develop this model, better known as blood group substance from human ovarian cyst fluid, has properties similar to those of human cell membrane glycoproteins (5).

In this approach, with the incorporation of pertinent controls, we observed nonspecific interactions of some lectins leading to false-positive signals. Our investigation of the source of falsepositive signals found that conventional blocking agents such as bovine serum albumin (BSA) and human serum albumin (HSA) are not suitable for accurate detection and identification of carbohydrate-binding proteins (CBP). These findings emphasized the need for a homogeneous sugar-free environment to maximize signal-to-noise ratio and enable accurate detection of sugars or glycans on macromolecules using CBPs.

The ideal blocking agent in solid phase assays after immobilization of glycans (6-9) or solid phase adsorption of glycoproteins $(10,11)$ has not been determined (12), and the choice of blocking reagent varies from one laboratory to another. Ideally a blocking reagent should not cross react with the CBP (6).
A

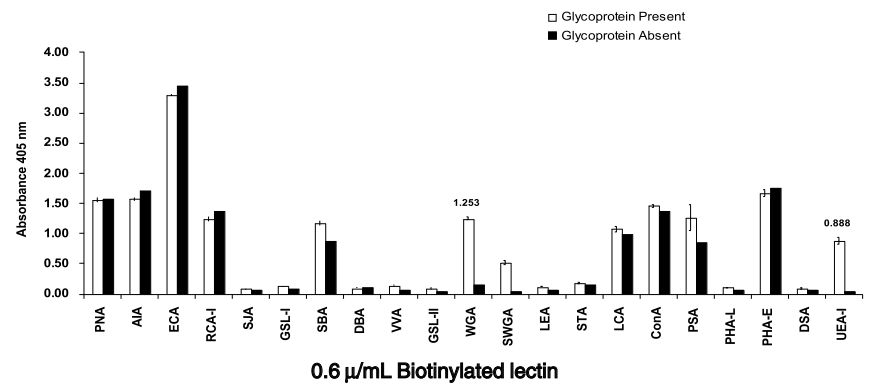

B

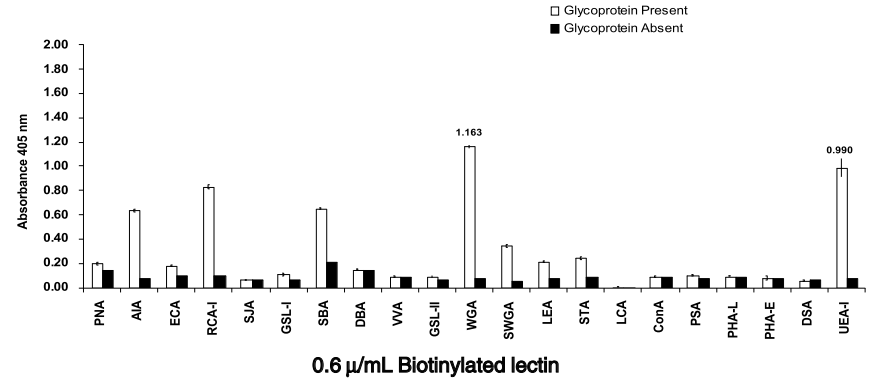

This problem is further amplified when attempting to screen for libraries of predefined or de novo CBP for defined complex sugar sequences.

The glycoprotein used as target in this model was prepared from human ovarian cyst fluid (1), and the serological activity (type $\mathrm{H}$ ) was determined using an anti-H immunoglobulin $\mathrm{M}$ (IgM) monoclonal antibody (data not shown). The serological activity along with the panel of plant lectins allows the testing of CBPs documented to bind blood group $\mathrm{H}, \mathrm{A}$, or B glycan determinants.

The assay parameters that we varied included the concentration of the glycoprotein and the blocking buffer conditions. The experiments were optimized using $0.6 \mu \mathrm{g} / \mathrm{mL}$ biotinylated Ulex europaeus agglutinin I (UEA-I). In the final version of the assay, the ELISA plates were coated with mucin glycoprotein overnight in $10 \mathrm{mM}$ phosphatebuffered saline (PBS) containing 150 $\mathrm{mM} \mathrm{NaCl}, \mathrm{pH} 7.2$, at $4^{\circ} \mathrm{C}$. PBS was chosen as a coating buffer as opposed to the conventional bicarbonate buffer (pH 9.8), as this buffer is better suited for the adsorption of the glycoprotein onto the solid phase Nunclon ${ }^{\circledR}$ Delta surface 96-well immunoplates (Nunc, Roskilde, Denmark) used in the assay. All subsequent steps were undertaken at room temperature. The wells containing glycoprotein were washed three times with PBS containing $0.05 \%$ w/v Tween ${ }^{\circledR} 20$ (PBS-T) and blocked to the brim for $2 \mathrm{~h}$ with either BSA (fraction V; Sigma-Aldrich, Dorset, UK) or ELISA Synblock from AbD

Figure 1. Comparative analysis of bovine serum albumin (BSA) and Synblock as blocking agent in testing binding specificity of mucin glycoprotein by 21 plant lectins. The result of probing the ovarian cyst fluid mucin glycoprotein with a panel of 21 lectins is shown. The glycoprotein was coated onto a 96 -well plate, blocked with either (A) $350 \mu \mathrm{L} 3 \% \mathrm{w} / \mathrm{v}$ BSA or (B) $350 \mu \mathrm{L}$ Synblock. The data are the mean result of three independent experiments, and error bars are the standard deviation of the mean. The biotinylated lectins were added at $0.6 \mu \mathrm{g} / \mathrm{mL}$, followed by $0.3 \mu \mathrm{g} /$ $\mathrm{mL}$ streptavidin-conjugated alkaline phosphatase, visualization with paranitrophenol phosphate for $30 \mathrm{~min}$, and signal quenching using 50 $\mu \mathrm{L} \mathrm{NaHCO}_{3}$. 


\section{Benchmarks}

Serotec (Oxford, UK). The wells were washed as before with PBS-T, and 100 $\mu \mathrm{L}$ each biotinylated lectin at $0.6 \mu \mathrm{g} / \mathrm{mL}$ (Vector Laboratories, Burlingame, CA, USA) in PBS were applied separately to each well and allowed to incubate for $1 \mathrm{~h}$. The plate was washed, and $100 \mu \mathrm{L}$ 1:3000 dilution of $1 \mathrm{mg} / \mathrm{mL}$ streptavidin conjugated to alkaline phosphatase (Vector Laboratories) were added for a further $1 \mathrm{~h}$, followed by a final wash, and signal was developed by incubation for $30 \mathrm{~min}$ with $100 \mu \mathrm{L} 1 \mathrm{mg} / \mathrm{mL}$ paranitrophenyl phosphate substrate (SigmaAldrich). The reaction was quenched using $50 \mu \mathrm{L} 3 \mathrm{M} \mathrm{NaHCO}_{3}$. As a negative control for each lectin, target mucin, the lectin, and alkaline phosphatase-conjugated streptavidin were systematically removed from the assay to monitor their contribution, if any, to the falsepositive signal. The presence of BSA as a blocking reagent was found to correlate with false-positive signals. Figure 1 shows the results of the experiments using either $3 \% \mathrm{w} / \mathrm{v}$ BSA or Synblock as the blocking reagent. The lectins that tested positive in the Synblock modification of the assay were Jacalin, soybean agglutinin, (SBA), wheat germ agglutinin (WGA), Ricinus communis agglutinin I (RCA-I), and UEA-I. These results correlate with both the monosaccharide composition of the glycoprotein (data not shown) and the known specificities of the lectins used in this assay (Table 1).

Gull and colleagues recently developed an ELISA-based method for quantification of WGA in serum in an application for lectin-mediated drug delivery (13). In this context, they used BSA as the blocking agent, and their assay worked well. We also found that the use of BSA as a blocking agent did not affect binding of either WGA or UEA-I; however, it is worthy of note that these were the only two lectins that produced a signal in our system when BSA was used as the blocking buffer. It was only when we changed the blocking buffer from BSA to Synblock that we were able to identify binding of other lectins to the mucin. Jacalin, RCA-I, and SBA were initially identified as false-positive results (Figure 1A) but were subsequently shown to be genuinely positive using the synthetic blocking reagent (Figure 1B). When HSA was used as a blocking reagent, false-positive results were also observed, although with different lectins (data not shown). Synblock, a buffered inert blocker is therefore an effective substitute for BSA as blocking agent and renders lectincarbohydrate interactions more specific.

\begin{tabular}{|c|c|c|}
\hline Lectins & Abbreviation & Sugar Specificity \\
\hline Peanut Agglutinin & PNA & Galactose \\
\hline Jacalin & AIA & Galactose/GalNAc \\
\hline Erythrina cristagalli & ECA & Galactose/GlcNAc \\
\hline Sophora japonica & SJA & GalNAc/Galactose \\
\hline Ricinus communis Agglutinin I & RCA-I & GalNAc/Galactose \\
\hline Griffonia simplicifolia Lectin I & GSL I & GalNAc/Galactose \\
\hline Soybean Agglutinin & SBA & GalNAc \\
\hline Dolichos biflorus & DBA & GalNAc \\
\hline Vicia villosa Lectin & VVA & GalNAc \\
\hline Griffonia simplicifolia Lectin II & GSL ॥ & GlcNAc \\
\hline Wheat Germ Agglutinin & WGA & GIcNAc/NANA \\
\hline Succinylated Wheat Germ Agglutinin & SWGA & GlcNAc \\
\hline Lycopersicon esculentum & LEA & GlcNAc \\
\hline Solanum tuberosum & STA & GlcNAc/Sialic acid \\
\hline Lens culinaris Agglutinin & LCA & Glucose/Mannose \\
\hline Concanavalin A & ConA & Glucose/Mannose \\
\hline Pisum sativum Agglutinin & PSA & Glucose/Mannose \\
\hline Phaseolus vulgaris Leucoagglutinin & PHA-L & Complex Sugar \\
\hline Phaseolus vulgaris Erythreoglubulin & PHA-E & Complex Sugar \\
\hline Datura stramonium & DSA & LacNAc \\
\hline Ulex europaeus Agglutinin I & UEA-I & Fucose \\
\hline
\end{tabular}

We think that Synblock provides two important properties, which are (i) effective blocking of exposed surface and (ii) environment homogeneity, which substantially improves binding assay data interpretation using this type of system.

The assay was extended to verify that the lectins were binding the mucin via carbohydrate-mediated interactions (14). To this end, the lectins were pre-incubated for $2 \mathrm{~h}$ with a range of monosaccharides prior to addition to the mucin (Figure 2). The inhibition tests performed using UEA-I demonstrate the specificity of this lectin where only 6-deoxy L-galactose (L-fucose), the terminal deoxy saccharide of the blood group $\mathrm{H}$ determinant (15), totally inhibited binding of the lectin. 2-deoxy D-galactose, which is an enantiomer of L-fucose, did not abrogate binding of UEA-I to glycoprotein. Inhibition of all the other lectins was achieved using a range of monosaccharides in a predictable manner (Figure 2, A-E). In all cases an appropriate inhibition is seen at lower monosaccharide concentrations matching the expected sugar recognition. However, in Figure 2, B, C, and $\mathrm{E}$, inhibition appears at high concentration for monosaccharides that do not correspond to the lectin specificities in these cases. This is an issue that further underlines the care needed in using this type of lectin assay. The inhibition data for the lectins that have had their corresponding sugar specificities documented in literature are in good agreement with monosaccharide inhibition concentrations.

In summary, we have optimized a method for determining protein-carbohydrate interactions using a panel of 21 lectins. Protein-carbohydrate interactions are notoriously weak and are modulated by a number of complex factors including glycan linkage, orientation, and structure. This modified ELISA method allows for precise interpretation of proteincarbohydrate interaction by decreasing the background signal obtained in the blocking systems commonly used. The increased interest in the study of both the proteome and glycome means that this simple methodology will have utility in a wide range of biological applications.

\section{ACKNOWLEDGMENTS}

This work was supported by The Guildford Bench Methodology Fund of The Biochemical Society, UK. B.A. 


\section{Benchmarks}

A
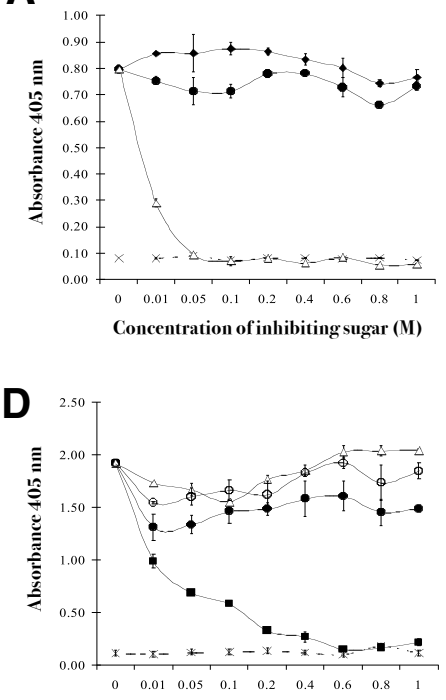

Concentration of inhibiting sugar (M)
B

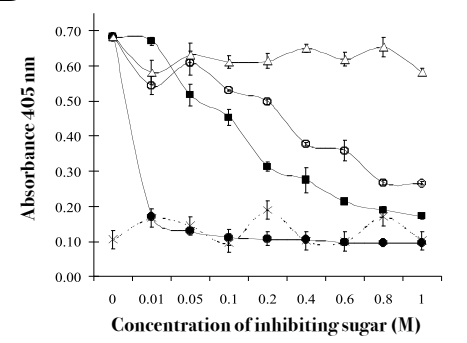

E

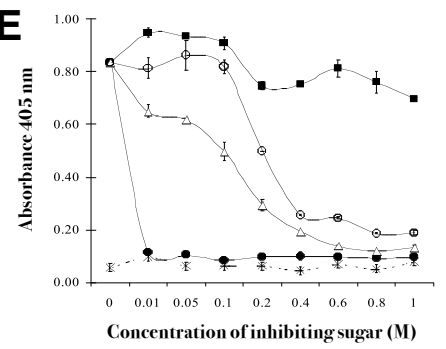

C

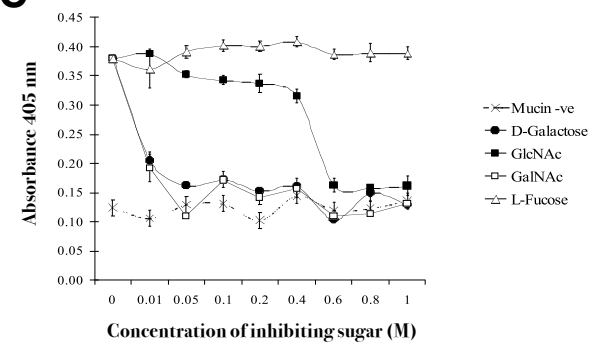

-D-Manno

$\times$ Mucin $-v \mathrm{c}$

Concentration of inhibiting sugar (M)

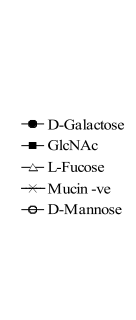

\section{- D-Galactose}

- GlcNAc

$\longleftarrow$ L-Fucose
$\times$ Mucin -ve

$\times$ Mucin-ve
$\rightarrow$ D-Mannose

Figure 2. Monosaccharide pre-inhibition of positive lectin binders to mucin glycoprotein as identified by the Synblock method. Lectin inhibition experiments using conditions from the Synblock-optimized model, the lectins (A) Ulex europaeus agglutinin I (UEA-I), (B) Jacalin, (C) soybean agglutinin (SBA), (D) wheat germ agglutinin (WGA), and (E) Ricinus communis agglutinin I (RCA-I) were pre-incubated with a range of concentrations of different monosaccharides coded in the legend by $(\Delta)$ L-fucose, $(\bullet)$ D-galactose, $(\diamond) \mathrm{D}$-fucose, $(\mathrm{O}) \mathrm{D}$-mannose, $(\boldsymbol{\square}) \mathrm{N}$-acetyl D-glucosamine $(\mathrm{GlcNAc})$, and (口) N-acetyl D-galactosamine (GalNAc) as shown. The results are mean absorbance values from three independent experiments, and error bars represent the standard deviation from the mean. The initial value (no inhibiting sugar) is the mean value of six experiments undertaken in the absence of inhibiting sugar. The negative control was carried out in the absence of glycoprotein represented as Mucin-ve.

was a recipient of a scholarship from the University of Westminster. We gratefully thank Professor Christopher Bucke and Dr. Ian J. Harmer for their comments and suggestions.

\section{COMPETING INTERESTS STATEMENT}

The authors declare no competing interests.

\section{REFERENCES}

1. Annison, E.F. and W.T. Morgan. 1952. Studies in immunochemistry. XI. The isolation and properties of the human blood-group H substance. Biochem. J. 52:247-258.

2. Engvall, E. and P. Perlman. 1971. Enzymelinked immunosorbent assay (ELISA). Quantitative assay of immunoglobulin G. Immunochemistry 8:871-874.

3. Damme, E.J.M.V. 1997. Handbook of Plant Lectins: Properties and Biomedical Applications. John Wiley \& Sons, New York.

4. Lis, H. and N. Sharon. 1998. Lectins: carbohydrate-specific proteins that mediate cellular recognition. Chem. Rev. 98:637-674.

5. Lloyd, K.O., B.W. Yin, P. Tempst, and H. Erdjument-Bromage. 2000. MUC-6 mucin is a major component of "blood group substance" from human ovarian cyst fluid. Biochim. Biophys. Acta 1474:410-414.

6. Houseman, B.T. and M. Mrksich. 2002. Carbohydrate arrays for the evaluation of protein binding and enzymatic modification. Chem. Biol. 9:443-454.

7. Ratner, D.M., E.W. Adams, J. Su, B.R. O'Keefe, M. Mrksich, and P.H. Seeberger. 2004. Probing protein-carbohydrate interactions with microarrays of synthetic oligosaccharides. ChemBioChem 5:379-382.

8. Smith, E.A., W.D. Thomas, L.L. Kiessling, and R.M. Corn. 2003. Surface plasmon resonance imaging studies of protein-carbohydrate interactions. J. Am. Chem. Soc. 125:6140-6148.

9. Shao, M.C. 1992. The use of streptavidin-biotinylglycans as a tool for characterization of oligosaccharide-binding specificity of lectin. Anal. Biochem. 205:77-82.

10. Eck, J., M. Langer, B. Mockel, K. Witthohn, H. Zinke, and H. Lentzen. 1999. Characterization of recombinant and plantderived mistletoe lectin and their B-chains. Eur. J. Biochem. 265:788-797.

11. Nomura, K., N. Takahashi, M. Hirose, S. Nakamura, and F. Yagi. 2005. Overall carbohydrate-binding properties of Castanea crenata agglutinin (CCA). Carbohydr. Res. 340:2004-2009.

12. Warkentin, P., I. Lundstrom, and $\mathbf{P}$. Tengvall. 1995. Protein-protein interactions affecting proteins at surfaces. Am Chem Soc Sym Ser 602:163-180.

13. Gull, I., M. Wirth, and F. Gabor. 2007. Development of a sensitive and reliable
ELISA for quantification of wheat germ agglutinin. J. Immunol. Methods 318:20-29.

14. Baker, D.A., S. Sugii, E.A. Kabat, R.M. Ratcliffe, P. Hermentin, and R.U. Lemieux. 1983. Immunochemical studies on the combining sites of Forssman hapten reactive hemagglutinins from Dolichos biflorus, Helix pomatia, and Wistaria floribunda. Biochemistry 22:2741-2750.

15. Watkins, W.M. and W.T. Morgan. 1952 Neutralization of the anti-H agglutinin in eel serum by simple sugars. Nature 169:825-826.

Received 4 May 2007; accepted 21 August 2007.

Address correspondence to Pamela Greenwell, Department of Biomedical Sciences, University of Westminster, 115 New Cavendish St, London, England, W1W 6UW,UK.e-mail: greenwp@wmin.ac.uk

To purchase reprints of this article, contact: Reprints@BioTechniques.com 